\title{
Quality of Life in Older Adults with Cochlear Implantation: Can It Be Equal to That of Healthy Older Adults?
}

\author{
Taskin Tokat ${ }^{1}$, Togay Müderris², Ergul Basaran Bozkurt² ${ }^{2}$ Uğurtan Ergun², \\ Abdulhalim Aysel $^{2}$, and Tolgahan Catli ${ }^{2}$ \\ ${ }^{1}$ Department of Otolaryngology-Head and Neck Surgery, Sakarya University Training and Research Hospital, Sakarya, Turkey \\ ${ }^{2}$ Department of Otolaryngology-Head and Neck Surgery, Bozyaka Training and Research Hospital, Izmir, Turkey
}

Received August 29, 2020

Revised January 15,2021

Accepted January 22, 2021

\author{
Address for correspondence \\ Taskin Tokat, MD \\ Department of Otolaryngology-Head \\ and Neck Surgery, \\ Sakarya University Training and \\ Research Hospital, \\ Adnan Menderes Street No. 195 \\ Adapazarı, Sakarya 54100, Turkey \\ Tel +90-505-385-56-06 \\ Fax +90-0-264-275-91-92 \\ E-mail tastokat@hotmail.com
}

Background and Objectives: This study aimed to evaluate the audiologic results after $\mathrm{co}-$ chlear implantation $(\mathrm{Cl})$ in older patients and the degree of improvement in their quality of life (QoL). Subjects and Methods: Patients over 65 years old who underwent $\mathrm{Cl}$ at implant center in Bozyaka Training and Research Hospital were included in this study ( $n=54 ; 34$ males and 20 females). The control group was patient over 65 years old with normal hearing ( $n=54 ; 34$ males and 20 females). We administered three questionnaires [World Health Organization Quality of Life-BREF (WHOQOL-BREF), World Health Organization Quality of Life-OLD (WHOQOL-OLD)], and Geriatric Depression Scale (GDS) to evaluate the QoL, Clrelated effects on activities of daily life, and social activities in all the subjects. Moreover, correlations between speech recognition and the QoL scores were evaluated. The duration of implant use and comorbidities were also examined as potential factors affecting QoL. Results: The patients had remarkable improvements (the mean score of postoperative speech perception $75.7 \%$ ) in speech perception after Cl. The scores for the WHOQOL-OLD and WHOQOL-BREF questionnaire responses were similar in both the study and control groups, except those for a two subdomains (social relations and social participation). The patients with longer-term $\mathrm{Cl}$ had higher scores than those with short-term $\mathrm{Cl}$ use. In general, the changes in GDS scores were not significant $(p<0.05)$. Conclusions: The treatment of hearing loss with $\mathrm{Cl}$ conferred significant improvement in patient's $\mathrm{QoL}(p<0.01)$. The evaluation of $\mathrm{QoL}$ can provide multidimensional insights into a geriatric patient's progress and, therefore, should be considered by audiologists.

J Audiol Otol 2021;25(3):138-145

KEY WORDS: Cochlear implantation · Quality of Life · Older adults · Hearing loss . Speech perception.

\section{Introduction}

Given the unique world of sound, it is acceptable that hearing loss is far beyond being only a sense disorder. Hearing loss affects speech perception and speech production, and negatively impacts on social communication, relationships, and employment, and as a result, the individual's quality of life (QoL) [1]. The daily life activities of older adults are increasing and furthermore, their request of participating in so-

This is an Open Access article distributed under the terms of the Creative Commons Attribution Non-Commercial License (https://creativecommons.org/licenses/by-nc/4.0/) which permits unrestricted non-commercial use, distribution, and reproduction in any medium, provided the original work is properly cited. cial activities leads to a higher discussion of how to treat hearing loss [2]. Morever, current clinical studies have shown that hearing loss is associated with cognitive decline and decreases in the QoL [3].

Although contemporary hearing aids are equipped with advanced technology to restore hearing loss, only cochlear implants (CIs) can provide enough stimulation for old patients with severe or profound hearing loss [4]. Evaluations of the effectiveness of $\mathrm{CI}$ in the older adults focused primarily on audiologic tests. However, the value of technological innovation is defined by the positive contribution in daily life. Therefore, recent studies emphasized that $\mathrm{CI}$ is necessary to restore hearing ability and improve social activities, cognitive 
ability, and QoL $[5,6]$. The appearing of the in light of this studies, the important question is "How much can CI contribute to QoL in old patients?" Recently published studies investigated the benefits of $\mathrm{CI}$ in old patients and analysed the correlation between QoL and speech perception, and demonstrated that it was necessary to know about QoL in CI users to achieve the most effective rehabilitation [7,8]. The aim of this study was to evaluate the impact of CI on QoL in geriatric patients with $\mathrm{CI}$ and to determine whether quality of their life improved to the level of the healthy and normal-hearing older adults population.

\section{Subjects and Methods}

This study included patients aged over 65 years at the time of CI and who had been followed up for at least 12 months in cochlear implat center in the Bozyaka Training and Research Hospital, Izmir, Turkey. The retrospective study protocol was approved by the ethics committee of the Bozyaka Training and Research Hospital and informed consents were obtained from all participants. All patients included to the study had undergone an appropriately comprehensive audiological evaluation prior to surgery. High-resolution computed tomography and magnetic resonance imaging of the temporal bone of the patients were performed in order to clarify the inner ear anatomy. The patients' medical records were reviewed for the etiology of the hearing loss, comorbidities, and audiological outcomes. Surgical records were reviewed to determine the technique of implantation, the extent of electrode array insertion (complete/partial), and intraoperative complications. The control group comprised of patients with bilateral normal hearing without any health problems, and aged over 65 years. Patients with severe medical problems or with severe cognitive impairments as proven with Mini-Mental State evaluation scores, which were assessed by a psychiatrist, were excluded from the study.

\section{Audiological assessment}

Pure tone audiometry was performed by an experienced audiologist the Bozyaka Training and Research Hospital, Izmir, Turkey. Speech recognition was assessed using open-set Turkish monosyllabic words. In audiometric tests after surgery was used only to the study group. All tests were performed in a quiet room using a live voice presented at $70 \mathrm{~dB}$.

\section{QoL assessment questionnaires}

In this study, QoL was assessed using the World Health Organization Quality of Life-OLD (WHOQOL-OLD), the World Health Organization Quality of Life-BREF (WHOQOL-
BREF), and Geriatric Depression Scale (GDS) [9-11]. These questionnaires have been validated in Turkish in a multicenter study [12-14].

The WHOQOL-OLD is specific to old people and uses a general scaling to evaluate patients' life by multi-directional way. The questionnaire is composed of 24 items that explore six dimensions: sensory abilities (SAB), autonomy (AUT), past present and future activities (PPF), social participation (SP), death and dying (DD), and intimacy (IN). The replies of the participant to the questions are rated on a five-point Likert-scale ( $1=$ very poor to $5=$ very good).

The WHOQOL-BREF is a shorter version of the original questionnaire (WHOQOL) designed to use more practical in clinical studies. The WHOQOL-BREF questionnaire comprised of 26 items and four dimensions, which measure the broad domains of physical health (PHH), psychological health (PSH), social relationships (SR), and environment (EN). Any question is scored on a five-point scale, with higher scores indicating better QoL.

The GDS consists of 30 items that measure depressive symptoms; answers are reported on a yes/no scale with high scores indicating more severe depression. Psychological evaluations of the patients were performed by the same psychiatrist who worked in the Bozyaka Training and Research Hospital.

\section{Statistical analysis}

Statistical analysis of the data was conducted using the Statistical Package for the Social Sciences 21.0.0 software package (IBM Corp., Armonk, NY, USA). The results are defined as mean \pm standard deviation (SD), median (minimummaximum), number (n), and percentage (\%). The data were analyzed to test the conformity to normal distribution by using the Kolmogorov-Smirnov and Shapiro-Wilk test. The ttest was performed for the analysis of normally distributed continuous variables. The Mann-Whitney U test and Kruskal-Wallis test were performed for the analysis of non-normally distributed continuous variables. The distribution of categorical variables was compared between the groups using the Pearson's Chi-square and Fisher's exact tests. $p$-values $<$ 0.05 were considered as statistically significant.

\section{Results}

The study group was consisted of 54 patients (34 males and 20 females) who completed the given questionnaires. The mean age in this group at the time of implantation was $71.32 \pm$ 1.6 (range, 65-79) years. The control group was 54 participants with normal hearing. The mean age of the participants in the control group was $70.3 \pm 1.8$ (range, $65-80$ ) years. No sta- 
tistically significant association in terms of sociodemographic variables (age, sex, and educational level) was found between the groups.

\section{Auditory performance}

The mean pre-operative hearing threshold in the patients with hearing loss (pure tone audiometry for the frequencies $0.5,1,2,4 \mathrm{kHz}$ ) was $108.7 \mathrm{~dB}$ (range, $85-125 \mathrm{~dB}$ ). The mean pre-operative speech perception score in quiet recognition was $24.57 \%$ (range, $0-65 \%$ ). Audiometric evaluation was obtained at least six months after CI activation. The mean pure tone audiometry (for the frequencies $0.5,1,2,4 \mathrm{kHz}$ ) in free field with the speech processor or with bimodal stimulation (depending on the modality normally used by the patients), was $33.1 \mathrm{~dB}$ (range, 20-50 dB). In terms of speech perception, the mean postoperative open-set word recognition score in quiet recognition was 75.7\% (range, 39-95\%). Speech perception and pure tone audiometry results were significantly improved compared with preoperative scores $(p<0.01)$. The duration of implant use in the study group ranged from 3 to 9 years (mean, $4.85 \pm 1.6$ years). Seven patients received Nucleus devices (Cochlear Corporation, Sydney, Australia), 23 pa-

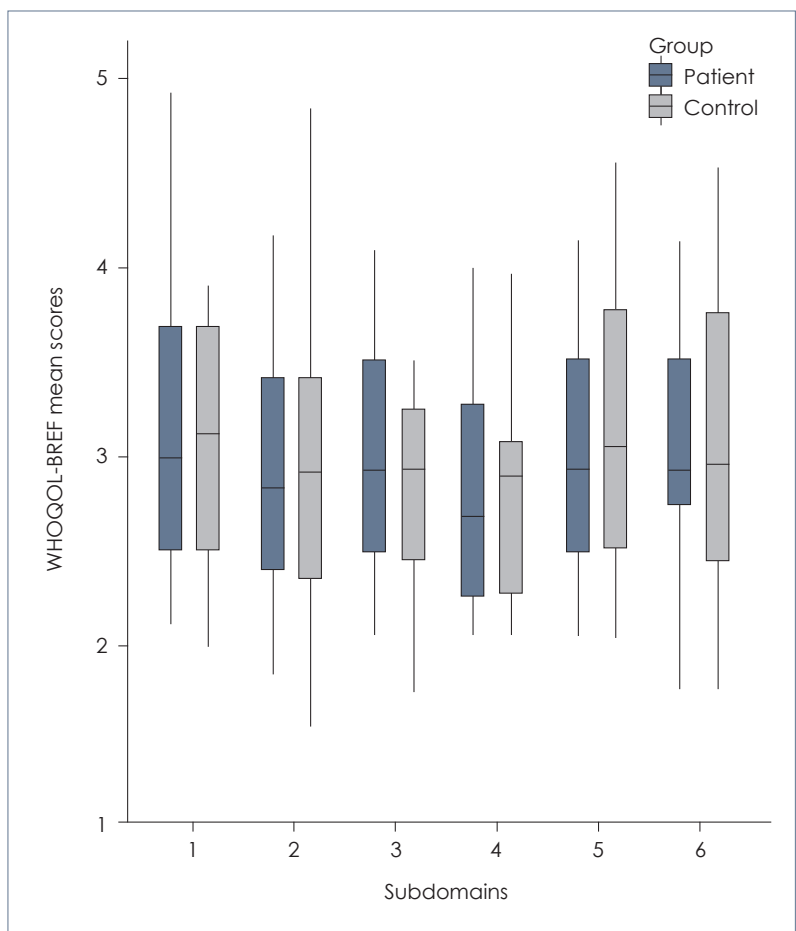

Fig. 1. WHOQOL-BREF questionnaire results: Correlations between patients and normal hearing individuals in the subdomains 1-5 (1: Environment, 2: Social relations, 3: General health, 4: Physical health, 5: Psychological health, 6: WHOQOL-BREF statistically outcome) and in the total score $(p<0.05)$. The line through the box is the mean scores. WHOQOL-BREF: World Health Organization Quality of Life-BREF. tients were implanted with Med-El devices (Medical Electronics, Innsbruck, Austria), 18 patients received Advanced Bionics Corporation (Advanced Bionics Corporation, Southern California, CA, USA), and six patients received Oticon devices (Oticon Medical, Chemin Saint Bernard, France).

\section{QoL assessment outcomes}

The normal-hearing old people had a tendency to have better QoL scores than the CI users, especially in the "physical health, psychological health, and social relations" subdomains in WHOQOL-BREF (Fig. 1) and also "social participation and past, present and future activities" subdomains in WHOQOL-OLD (Fig. 2).

Nevertheless, no significant difference was observed between the groups. Table 1 shows the correlations of questionnaire outcomes between the study group and control group. The global analysis of the WHOQOL-OLD and WHOQOLBREF answers showed an increase tendency parallel with the development of speech recognition, but this was marginally significant.

When the correlation between the duration of implant use and QoL was analyzed, the patients with longer-term CI use

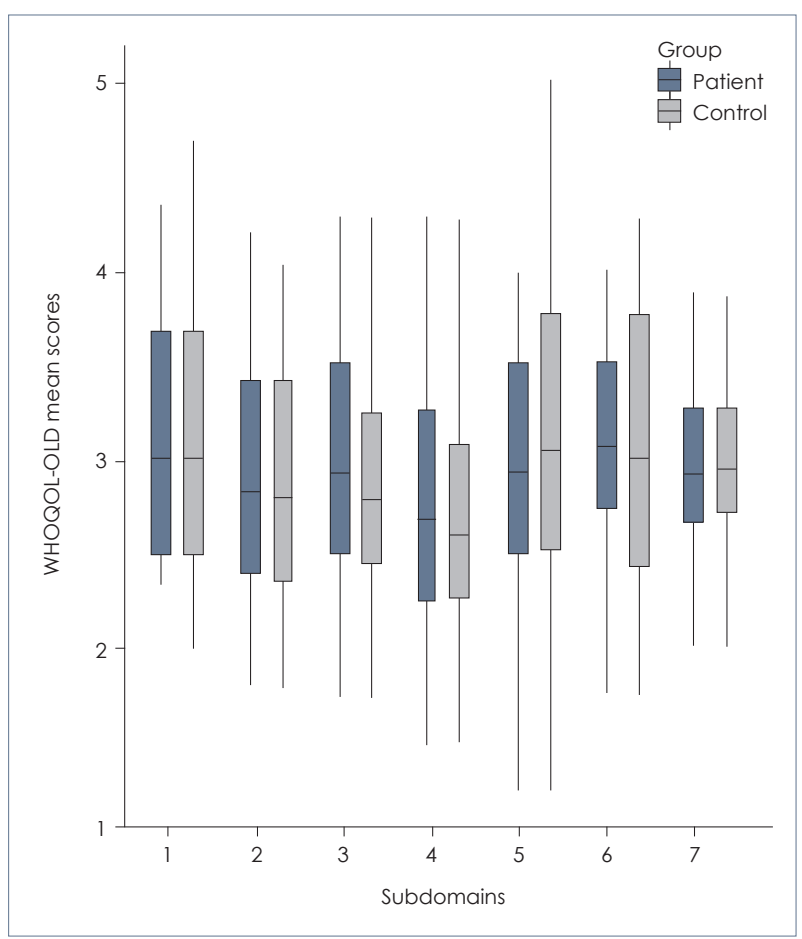

Fig. 2. Mean scores obtained by patients and normal hearing individuals in WHOQOL-OLD questionnaire (subdomains; 1 : Sensory abilities, 2: Autonomy, 3: Past, present and future activities, 4: Social participation, 5: Death and dying, 6: Intimacy, 7: WHOQOLOLD statistically outcome). WHOQOL-OLD: World Health Organization Quality of Life-OLD. 
Tokat $\mathrm{T}$, et al.

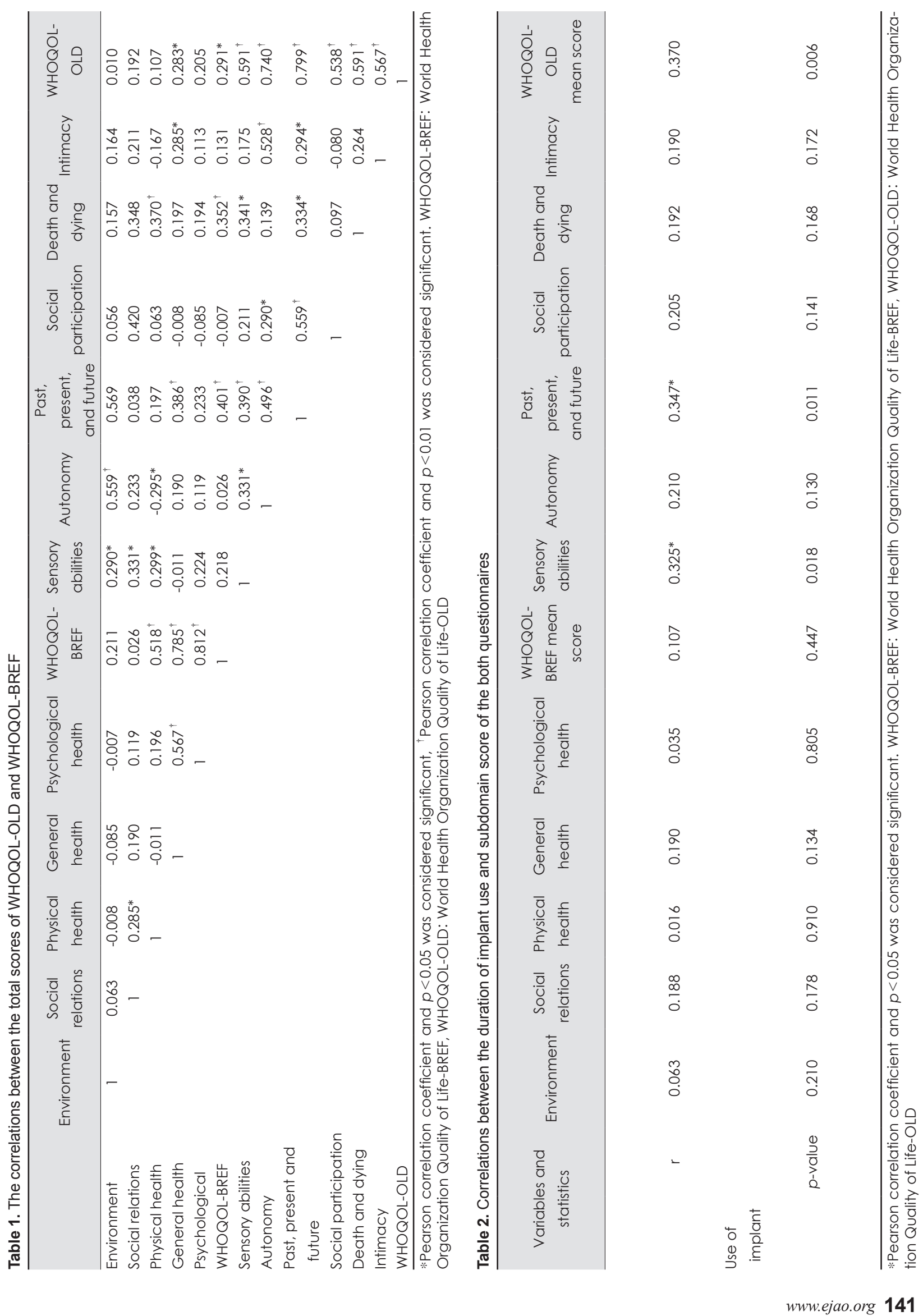


were determined to trend to higher scores, particularly in the subdomains of sensory abilities and past, present and future activities. While the correlation between the duration of implant use and WHOQOL-BREF was not statistically significant $(p<0.05)$, the correlation with WHOQOL-OLD showed significant difference $(p=0.006)$ (Table 2$)$. It was noticed that advanced chronologic age is not a major factor that limits the effectiveness of CI $(p<0.05)$.

The male patients in the study scored slightly higher (better QoL) than females in all WHOQOL-BREF subdomains except social relations and the WHOQOL-OLD, but the differences were not statistically significant $(p<0.05)$. "How would you rate your quality of life" is one of the key questions for WHOQOL-BREF. The mean score of this question was calculated as 2.74 for the patients with CI and 2.94 for the control group. Both mean scores were parallel with the WHOQOLOLD and WHOQOL-BREF mean scores, 2.91 and 2.96, respectively (Fig. 3).

The general health subdomain showed the highest correlation between the study and control groups in WHOQOLBREF (mean: $2.95 \pm 0.41,2.96 \pm 0.42$, respectively). Additionally, sequential linear regression analysis demonstrated that the psychological subdomain was the frailest component that had an effect on all QoL subdomains, after controlling for

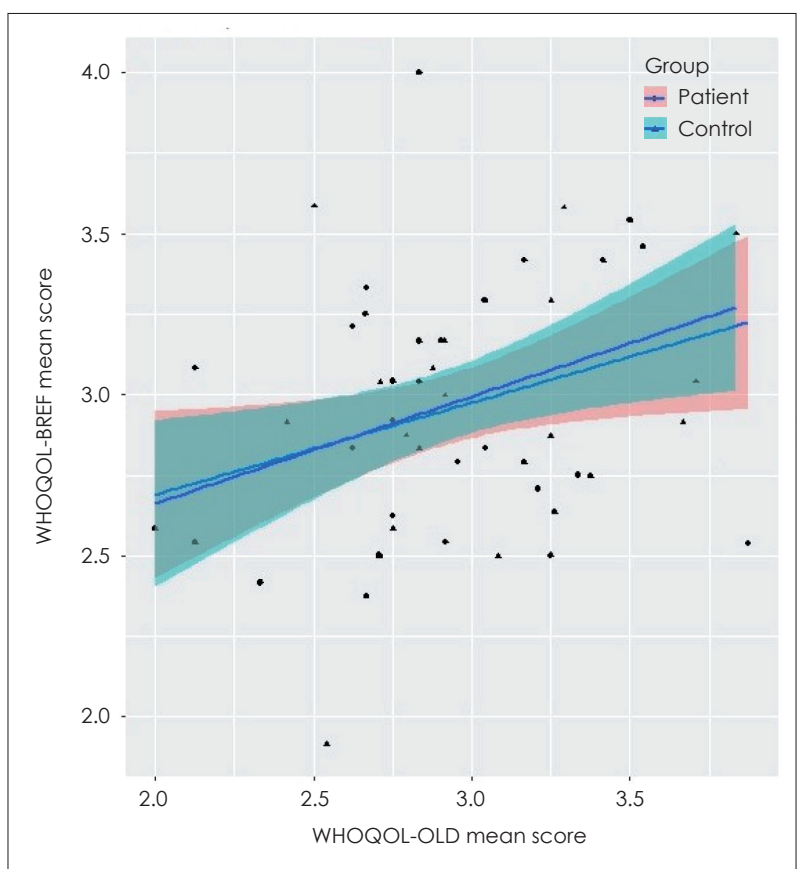

Fig. 3. The correlations between the mean scores of WHOQOLOLD and WHOQOL-BREF questionnaire. Chart type; scatter plot with correlation. Colored areas; $95 \%$ confidence interval. Black dots; intersection points. WHOQOL-BREF: World Health Organization Quality of Life-BREF, WHOQOL-OLD: World Health Organization Quality of Life-OLD. all other effects. Among all patients included in the study, lower age was more highly associated with autonomy and social participation subdomains. However, older age was associated more with the death and dying subdomain. The question, "Rate sensory functioning" in the sensory abilities subdomain was responded to as "very dissatisfied" by three patients of the CI group, 18 responded "dissatisfied," 18 responded as "neither satisfied nor dissatisfied," 10 as "satisfied," and five responded as "very satisfied." The mean scores for this question in the study group and control group were calculated as 2.96 and 2.90 , respectively.

The GDS scores of all patients in the study were transformed into percentages. In general, the changes in GDS scores were not significant $(p<0.05)$. Furthermore, the psychological dimension validity was supported by significant positive correlations between all domains of the WHOQOL-BREF and WHOQOL-OLD total scores and by strong correlations between the WHOQOL-BREF psychological subdomain and WHOQOL-OLD with GDS-30.

\section{Discussion}

The general opinion from basic hearing research is that $\mathrm{CI}$ is the most effective treatment modality in old patients with profound hearing loss. Given that CI can provide adequate functional gain and hearing restoration of hearing-impaired older patients, through improved speech perception, it is reasonable to consider that it might also improve QoL.

It is reasonable to attribute greater importance to CI performance in this context. Age-related striatal degeneration and loss of spiral ganglion neurons were evidenced in both animal and human studies [15]. Neuropsychological changes related to aging would impair CI performance and produce an increase in hearing thresholds. In a study that supported this hypothesis, Vermeire, et al. [16], against an overall improvement for both groups, reported better results in younger adults than in older patients in terms of speech perception. By contrast, Carlson, et al. [17] reported that patients implanted at an advanced age achieved the results of speech perception similar to those of younger adult patients. In a study conducted recently, Garcia-Iza, et al. [18] found no significant differences in recognition scores in silence between young and older adults. However, these measurements remain unsatisfactory to reflect the ultimate impact on daily life in old patients. Evaluating QoL besides auditory thresholds after implantation may lead a better guidance and motivation for patients. By definition, QoL is an "individual's perception of their position in life in terms of the culture and value systems in which they have social activities, and in relation to their goals, expectations, con- 
cerns and relations". Inevitably, the fundamental indicator for CI performance is to change in a positive direction the QoL of patients with CI. An important question in this context is whether the QoL in older patients with CI can be expected to be as good as that of old people with normal hearing.

The request to answer the aforementioned question has led us to design the present study which evaluate the impact on QoL of CI in older patients using two notionally different questionnaires that assess different domains of daily life. Choosing a questionnaire for the evaluation of QoL in the geriatric population is not easy because there is a wide variation in individual performance and perception, which is difficult to explain.

The Nijmegen Cochlear Implant Questionnaire (NCIQ) is specific to CI users, but its limitation could be that the sensory rehabilitation is the focal point and does not allow to evaluate all dimensions that would potentially relate to QoL in the older population. The SF-36 is not specific to old people and addresses generic health-related issues; therefore a lack of sensitivity of the SF-36 in evaluating changes in Hearing-related Quality of Life (HRQoL) after CI was also reported in previous studies [19-21]. The WHOQOL-OLD questionnaire was specifically adapted for old patients. WHOQOL-BREF provides reliable and valid results and has been widely used for assessing many diseases and conditions. The expanding of the cross-dimension approach to developing a measure means that it can be better compare and interpret between data from different designed groups.

In the past, a series of studies proved that aural rehabilitation through CI increased cognitive performance and counteracted the cognitive decline in the aging population [22]. Lin, et al. [23] stated that hearing loss impaired social relationships, leading to loneliness and degraded QoL in old people. Similarly, Fellinger, et al. [24] used the WHOQOL-BREF questionnaire to assess HRQoL in individuals with prelingual deafness in their study and pointed to the possible feelings of insecurity and inferiority in deafness.

In present study, some significant correlations were determined between speech perception and social participants, social relations, and psychological domains. The increase in speech perception test scores triggered the positive changes in the scores of the mentioned domains. In a relevant study, Francis, et al. [25] reported that gains in HRQoL were attributed to increases in speech perception and related to emotional benefits after CI. This findings were also supported by Cohen, et al. [26] who reported that increased audiologic performance provided positive effectives in HRQoL. In parallel, we found positive correlations between the auditory performance and social relations, social participation, and psychological domains. However, in the analysis of relations between au- ditory performance and overall questionnaire outcomes, no statistically significant associations could be demonstrated. Duration of implant use and age at implantation are clinical parameters that may affect to speech-perception results and overall questionnaire outcomes. Current age or age at implantation were not directly linked to the questionnaires or audiologic performance results in our study. Hirschfelder, et al. [27] reported that significant associations were found between the NCIQ total score and duration of CI use. However, we only found significant associations between the duration of CI use and the social relation, physical, social participation, and psychological domains (Table 1).

Kobosko, et al. [28] investigated the role of psychological factors on patient satisfaction after CI. They reported that CI satisfaction correlated with psychological factors significantly, contrary the variables of potential influence such as speech perception scores, duration of deafness, duration of CI use, or sociodemographic factors [28]. Out study result also supports the results of Kobosko, et al. [28]. Our results from the multiple regression models indicated that symptoms of depression presented a strong correlation with all WHOQOLOLD subdomains. However, because most studies on QoL in the geriatric population are cross-sectional, the interaction between current living circumstances, cultural factors, patient's individual characteristics, environmental effects, and QoL are still unclear.

A remerkable finding of our study is that the analysis of QoL questionnaires between older patients who received CI and normal-hearing old people revealed no significant difference. This determination may be associated with the increase in hearing-related QoL in patients after CI, which is also in line with other studies. Olze, et al. [29] stated that the effect of CI on hearing-related QoL may be even beyond the quantitative increase measured by auditory performance tests. Another reason for these results may be the lower social activity and working needs depending on aging. In recent study, Forli, et al. [30], divided 107 patients age over 40 years into three subgroups according to age at implantation and evaluated the corelation between speech perception and QoL. Interestingly, they reported higher QoL scores in the group of patients with older age at implantation ( $>70$ years), when is ignored the results of speech perception.

In the light of our findings, the effect of CI on mental health, social relationships and the ability to carry out daily activities, is undoubtedly positive and allows to quality of life in which in the many areas of life substantially equivalent to normalhearing old people.

In conclusion, the influence of CI in geriatric patients starts with hearing restoration and continues with the im- 
provement of QoL, which is closely linked to physical, psychological, and social components. However, improvements in QoL after CI cannot be elucidated only by the enhancements to audiologic outcomes. QoL is multidimensional and should accordingly be evaluated comprehensively. On the other hand, the individual perception of QoL in older patients may be relatively more favorable than in younger people due to the lower expectations reflecting their lower social and working needs. Even if this state may have affected the outcomes of our study, it is clear that CI improves QoL and provides hearing rehabilitation in old patients, regardless of chronologic age.

\section{Acknowledgments}

None

\section{Conflicts of interest}

The authors have no financial conflicts of interest.

\section{Author Contributions}

Conceptualization: Taskin Tokat and Togay Müderris. Data curation: Taskin Tokat and Togay Müderris. Formal analysis: Uğurtan Ergun and Togay Müderris. Investigation: Ergul Basaran Bozkurt and Uğurtan Ergun. Methodology: Taskin Tokat. Project administration: Taskin Tokat. Resources: Ergul Basaran Bozkurt and Togay Müderris. Software: Tolgahan Catli. Supervision: Taskin Tokat. Validation: Abdulhalim Aysel. Visualization: Abdulhalim Aysel. Writing — original draft: Taskin Tokat and Togay Müderris. Writing-review \& editing: Taskin Tokat and Tolgahan Catli. Approval of final manuscript: all authors.

\section{ORCID iDs}

Taskin Tokat

Togay Müderris

https://orcid.org/0000-0002-4515-8288

htps://orcid.org/0000-0003-4014-8176

Ergul Basaran Bozkurt https://orcid.org/0000-0001-5108-8485

Uğurtan Ergun

https://orcid.org/0000-0003-4381-9131

Abdulhalim Aysel

https://orcid.org/0000-0001-8584-308X

Tolgahan Catli

\section{REFERENCES}

1) Lenarz M, Sönmez H, Joseph G, Büchner A, Lenarz T. Cochlear implant performance in geriatric patients. Laryngoscope 2012;122: 1361-5.

2) Yamasoba T, Lin FR, Someya S, Kashio A, Sakamoto T, Kondo K. Current concepts in age-related hearing loss: epidemiology and mechanistic pathways. Hear Res 2013;303:30-8.

3) Poissant SF, Beaudoin F, Huang J, Brodsky J, Lee DJ. Impact of cochlear implantation on speech understanding, depression, and loneliness in the elderly. J Otolaryngol Head Neck Surg 2008;37:488-94.

4) Di Nardo W, Anzivino R, Giannantonio S, Schinaia L, Paludetti G. The effects of cochlear implantation on quality of life in the elderly. Eur Arch Otorhinolaryngol 2014;271:65-73.

5) Olze H, Szczepek AJ, Haupt H, Förster U, Zirke N, Gräbel S, et al. Cochlear implantation has a positive influence on quality of life, tinnitus, and psychological comorbidity. Laryngoscope 2011;121:2220-7.

6) Djalilian HR, King TA, Smith SL, Levine SC. Cochlear implantation in the elderly: results and quality-of-life assessment. Ann Otol Rhinol Laryngol 2002;111:890-5.

7) Budenz CL, Cosetti MK, Coelho DH, Birenbaum B, Babb J, Waltzman $\mathrm{SB}$, et al. The effects of cochlear implantation on speech perception

in older adults. J Am Geriatr Soc 2011;59:446-53.

8) Ambert-Dahan E, Routier S, Marot L, Bouccara D, Sterkers O, Ferrary E, et al. Cognitive evaluation of cochlear implanted adults using CODEX and MoCA screening tests. Otol Neurotol 2017;38:e282-4.

9) The WHOQOL Group. The World Health Organization quality of life assessment (WHOQOL): position paper from the World Health Organization. Soc Sci Med 1995;41:1403-9.

10) The WHOQOL Group. Development of the World Health Organization WHOQOL-BREF quality of life assessment. Psychol Med 1998; 28:551-8.

11) Yesavage JA, Brink TL, Rose TL, Lum O, Huang V, Adey M, et al. Development and validation of a geriatric depression screening scale: a preliminary report. J Psychiatr Res 1982-1983;17:37-49.

12) Eser S, Saatli G, Eser E, Baydur H, Fidaner C. The reliability and validity of the Turkish version of the World Health Organization Quality of Life Instrument-Older Adults Module (WHOQOL-OLD). Turk Psikiyatri Derg 2010;21:37-48.

13) Eser E, Fidaner H, Fidaner C, Eser SY, Elbi H, Göker E. Psychometric properties of the WHOQOL-100 and WHOQOL-BREF. J Psychiatry Psychol Psychopharmacol 1999;7:23-40.

14) Ertan T, Eker E, Sar V. Reliability and validity of the geriatric depression scale in Turkish elderly population. Archives of Neuropsychiatry (Turkey) 1997;34:62-71.

15) Frisina RD, Ding B, Zhu X, Walton JP. Age-related hearing loss: prevention of threshold declines, cell loss and apoptosis in spiral ganglion neurons. Aging (Albany NY) 2016;8:2081-99.

16) Vermeire K, Brokx JP, Wuyts FL, Cochet E, Hofkens A, Van de Heyning PH. Quality-of-life benefit from cochlear implantation in the elderly. Otol Neurotol 2005;26:188-95.

17) Carlson ML, Breen JT, Gifford RH, Driscoll CL, Neff BA, Beatty $\mathrm{CW}$, et al. Cochlear implantation in the octogenarian and nonagenarian. Otol Neurotol 2010;31:1343-9.

18) Garcia-Iza L, Martinez Z, Ugarte A, Fernandez M, Altuna X. Cochlear implantation in the elderly: outcomes, long-term evolution, and predictive factors. Eur Arch Otorhinolaryngol 2018;275:913-22.

19) Damen GW, Beynon AJ, Krabbe PF, Mulder JJ, Mylanus EA. Cochlear implantation and quality of life in postlingually deaf adults: longterm follow-up. Otolaryngol Head Neck Surg 2007;136:597-604.

20) McRackan TR, Hand BN, Velozo CA, Dubno JR. Association of demographic and hearing-related factors with cochlear implant-related quality of life. JAMA Otolaryngol Head Neck Surg 2019;145:422-30.

21) Mosnier I, Bebear JP, Marx M, Fraysse B, Truy E, Lina-Granade G, et al. Improvement of cognitive function after cochlear implantation in elderly patients. JAMA Otolaryngol Head Neck Surg 2015;141: 442-50.

22) Sonnet MH, Montaut-Verient B, Niemier JY, Hoen M, Ribeyre L, Parietti-Winkler C. Cognitive abilities and quality of life after cochlear implantation in the elderly. Otol Neurotol 2017;38:e296-301.

23) Lin FR, Yaffe K, Xia J, Xue QL, Harris TB, Purchase-Helzner E, et al. Hearing loss and cognitive decline in older adults. JAMA Intern Med 2013;173:293-9.

24) Fellinger J, Holzinger D, Gerich J, Goldberg D. Mental distress and quality of life in the hard of hearing. Acta Psychiatr Scand 2007;115: 243-5.

25) Francis HW, Chee N, Yeagle J, Cheng A, Niparko JK. Impact of cochlear implants on the functional health status of older adults. Laryngoscope 2002;112(8 Pt 1):1482-8.

26) Cohen SM, Labadie RF, Dietrich MS, Haynes DS. Quality of life in hearing-impaired adults: the role of cochlear implants and hearing aids. Otolaryngol Head Neck Surg 2004;131:413-22.

27) Hirschfelder A, Gräbel S, Olze H. The impact of cochlear implantation on quality of life: the role of audiologic performance and variables. Otolaryngol Head Neck Surg 2008;138:357-62.

28) Kobosko J, Jedrzejczak WW, Pilka E, Pankowska A, Skarzynski H. Satisfaction with cochlear implants in postlingually deaf adults and 
Tokat T, et al.

its nonaudiological predictors: psychological distress, coping strategies, and self-esteem. Ear Hear 2015;36:605-18.

29) Olze H, Gräbel S, Förster U, Zirke N, Huhnd LE, Haupt H, et al. Elderly patients benefit from cochlear implantation regarding auditory rehabilitation, quality of life, tinnitus, and stress. Laryngoscope 2012;
122:196-203.

30) Forli F, Lazzerini F, Fortunato S, Bruschini L, Berrettini S. Cochlear implant in the elderly: results in terms of speech perception and quality of life. Audiol Neurotol 2019;24:77-83. 